Bond University

Research Repository

\title{
Disinhibition and reward sensitivity in relation to alcohol consumption by university undergraduates
}

Lyvers, Michael; Czerczyk, Cameron; Follent, Anna; Lodge, Phoebe

Published in:

Addiction Research and Theory

DOI:

$10.3109 / 16066350802404158$

Licence:

Other

Link to output in Bond University research repository.

Recommended citation(APA):

Lyvers, M., Czerczyk, C., Follent, A., \& Lodge, P. (2009). Disinhibition and reward sensitivity in relation to alcohol consumption by university undergraduates. Addiction Research and Theory, 17(6), 668-677. https://doi.org/10.3109/16066350802404158

\section{General rights}

Copyright and moral rights for the publications made accessible in the public portal are retained by the authors and/or other copyright owners and it is a condition of accessing publications that users recognise and abide by the legal requirements associated with these rights.

For more information, or if you believe that this document breaches copyright, please contact the Bond University research repository coordinator. 


\section{Bond University}

\section{ePublications@bond}

12-1-2009

\section{Disinhibition and reward sensitivity in relation to alcohol consumption by university undergraduates}

Michael Lyvers

Bond University, michael_lyvers@bond.edu.au

Cameron Czerczyk

Bond University

Anna Follent

Bond University

Phoebe Lodge

Bond University

Follow this and additional works at: http://epublications.bond.edu.au/hss_pubs

Part of the Cognition and Perception Commons

Recommended Citation

Michael Lyvers, Cameron Czerczyk, Anna Follent, and Phoebe Lodge. (2009) "Disinhibition and reward sensitivity in relation to alcohol consumption by university undergraduates" Addiction research and theory, 17 (6), 668-677.

http://epublications.bond.edu.au/hss_pubs/258

This Journal Article is brought to you by the Faculty of Humanities and Social Sciences at ePublications@bond. It has been accepted for inclusion in Humanities \& Social Sciences papers by an authorized administrator of ePublications@bond. For more information, please contact Bond University's 
Disinhibition and Reward Sensitivity in Relation to Alcohol Consumption by University Undergraduates

Michael Lyvers, Ph.D.

Cameron Czerczyk, Postgraduate Diploma of Psychology

Anna Follent, Postgraduate Diploma of Psychology

Phoebe Lodge, Postgraduate Diploma of Psychology

Department of Psychology

Bond University

Gold Coast, Qld 4229 Australia 


\begin{abstract}
Deficits of prefrontal cortex functioning and associated executive cognitive impairments are well known correlates of chronic alcoholism and may reflect cumulative effects of high alcohol exposure. However, such associations may also reflect traits predating alcohol exposure which predispose to heavy drinking. In the present investigation, 60 university undergraduates aged 1825 years were administered the Alcohol Use Disorders Identification Test (AUDIT), Frontal Systems Behavior Scale (FrSBe), and Sensitivity to Punishment and Sensitivity to Reward Questionnaire (SPSRQ). All participants reported they were at least occasional drinkers who rarely or never used illicit drugs and had no reported history of head injury or neurological problems. All were sober at time of testing. AUDIT total scores were positively correlated with both FrSBe Disinhibition scores and SPSRQ Reward Sensitivity scores. The latter were negatively correlated with age at onset of regular alcohol use. High risk drinkers (as defined by AUDIT) had higher FrSBe Disinhibition and SPSRQ Reward Sensitivity scores compared to low risk drinkers. Findings indicate that even in a highly selected subset of young adults undergraduates attending a prestigious private university - associations may be present between indices of prefrontal cortex dysfunction and alcohol consumption, perhaps reflecting traits that predispose to heavy drinking.
\end{abstract}


University students have a higher prevalence of alcohol use as well as alcohol use disorders (AUDs) than lay persons of the same age (Dawson, Grant, Stinson \& Chou, 2004; Langlet, Kypri \& Stevenson, 2003; McGee \& Kypri, 2004). Kypri, Cronin and Wright (2005) reported that among 18-23 year olds, university students were twice as likely as non-students to be classified on the Alcohol Use Disorders Identification Test (AUDIT; Saunders, Aasland, Babor, De La Fuente \& Grant, 1993) as hazardous drinkers (AUDIT score 8-15) and three times as likely to be classified as harmful drinkers (AUDIT score 16+). The distinction between university students and lay people of the same age in terms of alcohol use has been attributed to the social environment of university life (Casswell, Pledger \& Pratap, 2002; Hughes, Power \& Francis, 1992). For example, as students are often away from home and family, they may be more likely to consume alcohol due to peer influences and for social reasons (Karam et al., 2007; Williams \& Clark, 1998). However, in the presence of social pressure to drink, not all students drink at hazardous or harmful levels, suggesting that excessive use of alcohol may be mediated in part by underlying trait factors such as impulsivity and/or anxiety sensitivity (Hair \& Hampson, 2006; Loxton \& Dawe, 2001; Novak, Burgess, Clark, Zvolensky \& Brown, 2003; Schmidt, Buckner \& Keough, 2007; Zuckerman \& Kulman, 2000).

The relationship between alcohol consumption and anxiety is unclear in young adult samples, with contradictory findings reported (Comeau, Stewart \& Loba, 2001; Kambouropoulos \& Staiger, 2004; Novak et al., 2003). One view is that anxiety may prevent people from drinking heavily due to threats of punishment (e.g., health problems, hangovers; Cox \& Blount, 1998), whereas another view suggests that individuals high in anxiety sensitivity may drink as a form of anxiolysis (Kambouropoulos \& Staiger, 2004). Impulsivity appears to have more support, with impulsivity levels in adolescents reported to be a positive predictor of later alcohol problems 
(Cooper, 2002; Soloff, Lynch \& Moss, 2000). Dawe and Loxton (2004) suggest that impulsivity is not a homogeneous construct but involves two factors: reward sensitivity and rash impulsiveness. Reward sensitivity is the motivation triggered by cues associated with approach towards a reward, and appears to be the aspect of impulsivity that is linked to substance abuse (Franken \& Muris, 2006; Loxton \& Dawe, 2001). A few recent studies have thus examined alcohol consumption in relation to measures of reward sensitivity based on Gray's Reinforcement Sensitivity Theory (RST), such as the BIS/BAS scales (Carver \& White, 1994) where the BAS (Behavioral Activation System) scale is the index of sensitivity to reward (SR) and the BIS (Behavioral Inhibition System) scale is an index of sensitivity to punishment (SP; related to anxiety) (Corr, 2002). Loxton and Dawe (2001) reported that BAS but not BIS scores were related to alcohol use as measured by the AUDIT. Similarly, Pardo, Aguilar, Molinuevo and Torrubia (2007) reported a significant positive association between BAS scores and alcohol consumption. BAS scores were also negatively related to age at first alcohol drink, i.e., the younger the drinker was at their first drink the higher their BAS scores tended to be. Pardo et al. defined age of first drink as the first time an individual had a drink in the absence of their family. The present study instead asked when people began drinking in a regular way (defined as a minimum of three times a month) in the hope that this would more directly tap the hypothesized inverse relationship between SR and age at onset of drinking. The age at which the first drink is taken is likely to reflect situational factors, whereas the age at which regular drinking is initiated may be more sensitive to individual differences such as SR. Further, instead of the BIS/BAS scales the Sensitivity to Punishment and Sensitivity to Reward Questionnaire (SPSRQ; Torrubia, Avila, Molto \& Caseras, 2001) was used to measure SR and SP, as the SPSRQ was devised to be more in line with the theoretical underpinnings of RST. One aim of the current study was to 
examine the associations between drinking levels (as defined by the AUDIT) of university students and their levels of SR and SP as defined by the SPSRQ. Consistent with previous findings cited above for adult samples, in a university undergraduate sample SR scores were expected to be positively related to AUDIT scores and negatively related to age of onset of regular drinking. Given the previous negative findings on SP, we expected no such association of SP with AUDIT scores.

A second aim of the current study was more exploratory: to examine an index of everyday symptoms of frontal lobe dysfunction in relation to university student alcohol consumption as measured by AUDIT. Impairments in executive cognitive performance related to dysfunction of the frontal lobes have been well documented in chronic heavy drinkers and alcoholics (Cargiulo, 2007; Kokavec \& Crowe, 1999; Lyvers, 2000; Noel et al., 2001; Sullivan, Rosenbloom \& Pfefferbaum, 2000). Although alcoholics have been shown as a group to exhibit impaired executive cognitive functioning compared to non-alcoholic controls, such results do not necessarily tell us whether the deficits preceded the alcoholism or whether all such deficits emerged as a result of chronic exposure to neurotoxic effects of alcohol. Spinella (2003) supports a preceding role of frontal lobe dysfunction in addictions based on research conducted on people who suffered traumatic brain injury (TBI). Longitudinal studies of people with TBI indicate that an increase in substance abuse often follows brain injury. For example, Spinella cited evidence that $20 \%$ of individuals who initially abstained from alcohol or who were light drinkers progressed to high consumption levels after their injury. Fontaine et al. (1999) reported similar findings that $15 \%$ of TBI patients developed alcohol dependence problems within 2 years of their brain injury. Although these changes could be due to psychosocial stressors resulting from a TBI, they may, in part, be directly due to TBI-related frontal lobe dysfunction and resultant changes in 
emotional regulation and impulse control (Spinella, 2003). In further support for this hypothesis, studies conducted on children of alcoholics, who are at elevated risk for alcoholism, have reported that such children tend to show deficiencies on neuropsychological tests of executive cognitive functioning (Giancola et al., 1996). Both social drinking and family history of alcoholism have been related to neurocognitive functioning in non-alcoholic individuals (Alterman \& Hall, 1989). If present, inherited deficiencies in prefrontal cortical functioning are theoretically likely to be associated with corresponding deficits in impulse control and executive cognitive functioning, which may in turn predispose to alcoholism and other substance disorders.

The Frontal Systems Behaviour Scale (FrSBe; Grace \& Malloy, 2001) assesses several of the broad functions mediated by the frontal lobes as manifested in a brain injured patient's daily social and occupational functioning, and was used by Verdejo-Garcia, Rivas-Perez, LopezTorrecillas and Perez-Garcia (2006) to examine frontal lobe related deficits associated with other drug addictions. Three dimensions of frontal lobe dysfunction are examined by the FrSBe: executive dysfunction (e.g., cognitive and behavioural disorganisation, loss of hypothesis generation and testing, impaired problem solving and working memory), apathy (e.g., loss of spontaneity, drive and curiosity), and disinhibition (e.g., impulsivity, aggression, loss of foresight, and inability to delay gratification). These three subscales were designed to detect behavioural signs of disruption to three circuits connecting the prefrontal cortex with subcortical areas: the anterior cingulate circuit (damage associated with apathy), the orbitofrontal circuit (damage associated with disinhibition), and the dorsolateral circuit (damage associated with executive dysfunction). In the present study, the FrSBe was administered to university students who were not chronic alcoholics and thus were unlikely to have suffered from brain damage due to chronic exposure to neurotoxic levels of alcohol. Thus if FrSBe scores were found to be 
positively related to alcohol consumption in this sample, such a finding would be consistent with the notion that inherent deficiencies of frontal lobe functioning predispose to heavier alcohol intake. Spinella (2003) reported associations between scores on the FrSBe Disinhibition scale and use of tobacco and illicit drugs. As orbitofrontal dysfunction has been specifically linked to chronic alcoholism in previous work (e.g., Modell \& Mountz, 1995), and chronic alcoholism as noted above has been linked with executive cognitive deficits, we hypothesised that FrSBe Disinhibition and Executive Dysfunction scores would be positively related to alcohol consumption as well as SR in a university sample. Although no specific interactions with gender were anticipated, participant gender was a factor in the analyses for exploratory purposes.

\section{Method}

\section{Participants}

Sixty undergraduate university students, 39 females $(M=20.97 \mathrm{yr}, S D=1.98)$ and 21 males $(M=21.38 \mathrm{yr}, S D=2.09)$, attending Bond University, Queensland, Australia, completed the current study for introductory psychology course credit. Participants were recruited via signup sheets that provided a brief description of the study's procedures. The stated criteria excluded anyone who was intoxicated, had recently suffered from a head injury, used illicit drugs at least 3 times per month, did not drink alcoholic beverages at least occasionally, or was under the legal drinking age (in Australia) of 18. The study was approved by the Bond University Human Research Ethic Committee (BUHREC) and all participants read and signed an appropriate informed consent form prior to participating.

\section{Materials}

The Alcohol Use Disorders Identification Test (AUDIT; Saunders et al., 1993) is a self report measure widely used in screening for harmful and hazardous drinking in adolescent and 
adult populations. The AUDIT is comprised of 10 items answered on Likert scales. There are 3 questions assessing alcohol consumption (e.g., "How often do you have a drink containing alcohol?"), 3 assessing signs of alcohol dependence (e.g., "How often during the last year have you failed to do what was normally expected of you because of drinking?"), and 4 assessing alcohol-related problems (e.g., "Have you or someone else been injured because of your drinking?"). The AUDIT total score is devised by the summation of a participant's responses to all 10 items; possible scores range from 0-40. A score of 0-8 is indicative of "Low Risk," a score of $8-15$ suggests the respondent is drinking at a "Hazardous" level, and a score of 16 or higher suggests the respondent is drinking at a "Harmful" level. The AUDIT has excellent internal reliability and good to excellent test-retest reliability (Kane, Loxton, Staiger \& Dawe, 2004; Reinert \& Allen, 2007; Rubin et al., 2006). Recent studies have shown that the AUDIT demonstrates construct, discriminate and concurrent validity (Bergman \& Kallmen, 2002; Shields \& Caruso, 2004).

The Sensitivity to Punishment and Sensitivity to Reward Questionnaire (SPSRQ; Torrubia, Avila, Molto \& Caseras, 2001) is a self report measure assessing a participant's appetitive (SR) and aversive (SP) motivational system functioning levels in adolescent and adult populations. The SPSRQ is comprised of 48 items, of which 24 assess SR and 24 assess SP. Participants respond to all questions with either a yes or no response, with affirmative responses being summated to form SR and SP scores. The SR and the SP scales are reported to show very good internal consistency and test-retest reliability as well as convergent, construct and discriminate validity (Caseras, Avila, \& Torrubia, 2003; Sava \& Sperneac, 2006).

The Frontal Systems Behaviour Scale (FrSBe) was designed to measure behaviour associated with damage to the frontal lobes (Davis \& Tremont, 2007; Grace \& Malloy, 2001; 
Malloy \& Boyle, 2005). The FrSBe was originally designed for neurological patients, with preillness/injury and post-illness/injury ratings taken from the patient as well as family members or caretakers. The original form of the scale asks for pre- and post-injury ratings from the participant and others, but as the present study was conducted in a student rather than clinical sample, participants were asked to provide only current ratings of themselves (Spinella, 2003). The FrSBe provides a measure of three frontal behavioural syndromes as manifested in everyday life: apathy, disinhibition and executive dysfunction. There are 46 items with each item rated on a 5-point Likert scale where 1 = Almost never, 2 = Seldom, 3 = Sometimes, $4=$ Frequently, and $5=$ Almost always. Sample items include "Repeat certain actions or get stuck on certain ideas" and "Show poor judgement, poor problem solver." There are 14 reverse-scored items, including "Use strategies to remember important things (for example, write notes to myself)" and "Am able to plan ahead." Scoring yields scores on the three subscales of Apathy (14 items), Disinhibition (15 items), and Executive Dysfunction (17 items) as well as a Total score. High internal consistency is reported for the Total score as well as for each subscale score (Grace \& Malloy, 2001) and good convergent validity with other neuropsychological measures is reported as well (Norton, Malloy \& Salloway, 2001).

Finally, demographic information was obtained using a self report questionnaire comprised of 14 items. Questions pertained to age at onset of regular drinking (AOD), defined as age when first began drinking three or more times per month; current age, gender, illicit drug use (no participant reported current use), and smoking (only 3 participants reported that they smoked). 


\section{Procedure}

All participants were de-identified upon consenting to the study and were given a unique code which acted as their only form of identification. Participants completed a questionnaire battery comprised of the AUDIT, SPSRQ, FrSBe and demographic questions. The questionnaires were administered in a quiet room to a maximum of four participants at any one time. All instructions were clearly specified on each questionnaire, however the researchers also verbally read out the instructions as well. Each participant completed the questionnaires in a varied sequence, as the order was counterbalanced. In total the questionnaires took about $10 \mathrm{~min}$ to complete.

\section{Results}

Intercorrelations were calculated among AUDIT measures (Consumption, Dependence, Alcohol Related Problems and Total scores), SPSRQ SR and SP scores, FrSBe measures (Apathy, Disinhibition, Executive Dysfunction and Total scores), and AOD (see Table 1). The important correlations to note in Table 1 are (1) the strong positive correlations of SR with all AUDIT measures, (2) the significant positive correlations of SR with FrSBe Disinhibition, Executive Dysfunction and Total scores, and (3) the significant negative correlation of SR with AOD, all consistent with predictions. By contrast, SP was not correlated with AUDIT but was positively correlated with FrSBe Apathy and Executive Dysfunction as well as FrSBe Total score, which were unanticipated findings.

Individual differences in SR and SP related to alcohol use by university students were further examined by dividing participants into three groups based on their AUDIT Total scores following guidelines described previously above for this measure: low risk (total score $0-7, n=$ 
20), hazardous (total score $8-15, n=19$ ), and harmful (total score $16+, n=21$ ). Group differences on SPSRQ SR and SP were then assessed.

First, separate two-way (AUDIT group X gender) between groups analyses of variance (ANOVAs) were run on SR and SP due to the lack of correlation between these two dependent variables (Tabachnick \& Fidell, 2007). Prior to running the two-way ANOVA, Levene's test of equality of error variance was not significant, thus satisfying the assumption of homogeneity of variance. Box's test of equality of covariance matrices was greater than .001 , thus this assumption was also met. Preliminary analysis also revealed that the data were void of extreme outliers and showed normality; thus no cases were removed. The $2 \times 3$ between groups ANOVA on SR revealed a significant main effect of AUDIT group, $F(2,54)=12.14, p=.001, \eta^{2}=.31$, power $=.99$. Post-hoc comparisons using the Tukey HSD test revealed that the mean SR score for the harmful $(M=15.10, S D=3.73)$ and hazardous AUDIT groups $(M=12.79, S D=4.12)$ were significantly higher than that of the low risk group $(M=8.45, S D=3.14)$. The main effect of gender was also significant, $F(1,54)=6.51, p=.025, \eta^{2}=.11$, power $=.71$. Males scored significantly higher $(M=14.57, S D=4.77)$ than females $(M=10.85, S D=3.93)$ overall. There was no interaction between gender and alcohol consumption, $F(2,54)=.17, p=.84$.

A second $2 \times 3$ ANOVA was conducted on SP. Again all assumptions were satisfied prior to running the test. There were no significant main effects for gender, $F(1,54)=.09$, $p=.77$, or AUDIT group, $F(2,54)=1.53, p=.23$. There was also no interaction, $F(2,54)=$ $.01, p=.98$.

To examine group differences on FrSBe measures, a 2 (gender) x 3 (AUDIT group) between-groups multivariate analysis of variance (MANOVA) was performed. Three dependent variables were examined: FrSBe Apathy, Disinhibition, and Executive Dysfunction scores. 
Mahalanobis Distance revealed that 1 participant exceeded the critical chi squared value of 18.47 and thus was removed from further analysis. No univariate outliers were detected. The assumption of homogeneity of variance-covariance matrices (Box's M) was not significant, indicating no violation. The Levene's test was significant only for one of the dependent variables, Disinhibition; therefore the alpha level was set at a more conservative value of .01 (Tabachnick \& Fidell, 2007). Lastly, Mauchly's Test of Sphericity indicated that the assumption of sphericity was satisfied, spherical epsilon $=1.00$. After all assumptions were met, the MANOVA was conducted. Using Pillai's trace there was no effect of gender, $F(3,51)=2.13, p$ $=.11$, and no interaction, $F(6,104)=1.23, p=.30$. There was however a significant main effect of AUDIT group on the combined dependent variables using Pillai's Trace, $F(6,104)=3.08, p=$ $.004 ; \eta^{2}=.15$, power $=.90$. When the results for the dependent variables were considered separately, only Disinhibition was significant, $F(2,53)=7.01, p=.002, \eta^{2}=.21$, observed power $=.91$. Tukey post-test revealed that as predicted, low risk drinkers $(M=27.95, S D=5.53)$ scored significantly lower on Disinhibition than did hazardous $(M=32.68, S D=7.91)$ or harmful drinkers $(M=33.95, S D=6.78)$.

\section{Discussion}

The present university student sample showed high levels of drinking defined as hazardous or harmful by the AUDIT, with one-third of the sample scoring in the hazardous range and one-third scoring in the harmful range according to the criteria set by Saunders et al. (1993). The important findings of the present study were that (1) SR was significantly positively correlated with all AUDIT measures as well as with FrSBe Total score, Disnihibition and Executive Dysfunction, (2) SR was significantly negatively correlated with AOD, and (3) harmful and hazardous drinkers as defined by AUDIT scored significantly higher on SR and 
Disinhibition than did low risk drinkers. All of these findings were consistent with predictions based on previous work mostly done on older and/or alcoholic samples.

Consistent with the present findings, SR was previously suggested to be the component of impulsivity that promotes initiation of hazardous drinking patterns (Dawe et al., 2004); however, other factors may be responsible for the progression from hazardous to harmful drinking, as in the present study hazardous and harmful groups did not differ on SR. The hypothesis that AOD would be negatively related to SR was supported, thus giving support to the idea posited by Pardo et al. (2007) that individuals with higher levels of SR are likely to start consuming alcohol at an earlier age, perhaps leading to an increased risk of neurological damage in this time of cognitive vulnerability (Andersen, 2003; Dahl, 2004). Seemingly consistent with the latter interpretation, hazardous and harmful drinkers also scored significantly higher on the Disinhibition scale of the FrSBe than did drinkers classed as low risk. Disinhibition was positively correlated with all AUDIT measures, whereas Executive Dysfunction was positively correlated only with the Alcohol-Related Problems subscale. The Disinhibition scale is based on clinical observations of patients with lesions of orbitofrontal cortex, an area which plays a major role in reward and reinforcement (Spinella, 2003). Many effects of orbitofrontal damage (e.g., altered personality and social conduct, verbal and behavioral disinhibition) can be explained by an underlying change in responsiveness to reward contingencies. In the present context, disinhibition may promote the continuation of drinking once it has started and thus may play a part in the occurrence of binge drinking (Rose \& Duka, 2007).

As the present findings were obtained in young adult university students, the AUDIT group differences on Disinhibition are perhaps unlikely to reflect alcohol-related neurological damage but rather may indicate an inherent trait that promotes over-consumption of alcohol. The 
significant correlation between SR and FrSBe Disnihibition presumably reflects the overlap between these two constructs (Pardo et al., 2007), but this does not explain the significant correlation between SR and FrSBe Executive Dysfunction. There were also unanticipated correlations of SP with FrSBe Apathy and Executive Dysfunction. Conceivably the latter correlations might reflect motivational and cognitive impairments associated with anxiety, although that is entirely speculative until these unexpected associations can be replicated in a larger sample.

More generally, present findings are consistent with the theory of so-called Type II alcoholism, which is defined as having an early onset (before age 25) and is said to be characterized by impulsive and disinhibited personality traits. In contrast, there was no evidence supporting a relationship between SP and alcohol use. Note that Type I alcoholism, in contrast to Type II, is defined as having a late onset (after age 25) and is said to be related to anxiety sensitivity and neuroticism (Varma, Basu, Malhotra, Sharma \& Matoo, 1994). Perhaps the university student sample of the present study was too young for any evidence consistent with the etiology of Type I alcoholism to be evident, as anxiety tends to be a predominant factor in older samples (Mazas, Finn \& Steinmetz, 2000).

In conclusion, the present study has linked traits of SR and disinhibition to hazardous and harmful alcohol consumption by university students. These findings suggest that potentially dangerous levels of alcohol intake at university are not solely attributable to the social environment of university life, with its peer pressures. Rather, factors that individual students bring with them to university life also appear to play an important role. 
Table 1

Intercorrelations among AUDIT total score (ATOT), AUDIT Consumption score (Cons), AUDIT Dependence score (Dep), AUDIT Problems score (Probs), SPSRQ-SP score, SPSRQ-SR score, FrSBe-Apathy score (Apath), FrSBe-Disinhibition (Dis), FrSBe-Executive Dysfunction score (Exec), FrSBe Total score (FTOT), and age at onset of regular drinking (AOD).

\section{Dep Prob ATOT SP SR Apath Dis Exec FTOT AOD}

AUDIT:

\begin{tabular}{|c|c|c|c|c|c|c|c|c|c|c|}
\hline Cons & $.58 * *$ & $.56^{* *}$ & $.84 * *$ & -.02 & $.57 * *$ & -.07 & $.38 * *$ & .17 & .22 & $-.30 *$ \\
\hline Dep & & $.66^{* *}$ & $.82 * *$ & -.07 & $.48 * *$ & .03 & $.31 *$ & .12 & .23 & -.22 \\
\hline Prob & & & $.89 * *$ & -.02 & $.51 * *$ & -.04 & $.31 *$ & $.26^{*}$ & .21 & -.21 \\
\hline ATOT & & & & -.04 & $.60 * *$ & -.04 & $.39 * *$ & .23 & .25 & $-.29 *$ \\
\hline
\end{tabular}

SPSRQ

SP

$\begin{array}{lllll}.00 & .40 * * .03 & .41 * * & .34 * * & .03\end{array}$

SR

$.08 \quad .47 * * .28 * \quad .36 * *-.39 * *$

FrSBe

Apath

$.30 * \quad .70 * * \quad .80 * * \quad .07$

Dis

$.52 * * \quad .77 * *-.07$

Exec

$.85^{* *}-.09$

FTOT

$-.05$

AOD

$* \mathrm{p}<.05 ; * * \mathrm{p}<.01$ 


\section{References}

Alterman, A. I., \& Hall, J. G. (1989). Effects of social drinking and familial alcoholism risk on cognitive functioning: null findings. Alcoholism: Clinical and Experimental Research, 13(6), 799-803.

Andersen, S. (2003). Trajectories of brain development: Point of vulnerability or window of opportunity. Neuroscience and Biobehavioral Reviews, 27, 3-18.

Bergman, H., Kallmen, H. (2002). Alcohol use among Swedes and a psychometric evaluation of the Alcohol Use Disorders Identification Test. Alcohol, 37, 245-251.

Carver, C. S., \& White, T. L. (1994). Behavioural inhibition, behavioural activation, and affective responses to impending reward and punishment: The BIS/BAS scales. Journal of Personality and Social Psychology, 67, 319-333.

Cargiulo, T. (2007). Understanding the health impact of alcohol dependence. American Society of Health-System Pharmacists, 64(3), 1-17.

Caseras, X., Avila, C., \& Torrubia, R. (2003). The measurement of individual differences in behavioural inhibition and behavioural activation systems: A comparison of personality scales. Personality and Individual Differences, 34, 999-1013.

Casswell, S., Pledger, M., \& Pratap, S. (2002). Trajectories of drinking from 18 to 26 years: Identification and prediction. Addiction, 97, 1427-1437.

Comeau, N., Stewart, S. H., \& Loba, P. (2001). The relations of trait anxiety, anxiety sensitivity, and sensation seeking to adolescents motivations for alcohol, cigarette and marijuana use. Addictive Behaviours, 26, 803-825. 
Corr, P. J. (2002). J.A. Gray's Reinforcement Sensitivity Theory: Tests of the joint subsystems hypothesis of anxiety and impulsivity. Personality and Individual Differences, 33, 511 532.

Cox, W. M., \& Blount, J. P. (1998). Are punishment-avoidant college students protected from excessive alcohol consumption? Psychology of Addictive Behaviours, 12(4), 227-232.

Davis, J.D., \& Tremont, G. (2007). Impact of frontal systems behaviour functioning in dementia on caregiver burden. Neuropsychiatry Clinical Neuroscience, 19(1), 43-49.

Dawe, S., Gullo, M. J., \& Loxton, N. J. (2004). Reward drive and rash impulsiveness as dimensions of impulsivity: Implications for substance misuse. Addictive Behaviours, 29, 1389-1405.

Dawe, S., \& Loxton, N. J. (2004). The role of impulsivity in the development of substance use and eating disorders. Neuroscience and Biobehavioral Reviews, 28, 343-351.

Dawson, D. A., Grant, B. F., Stinson, F. S., \& Chou, P. S. (2004). Another look at heavy episodic drinking and alcohol use disorders among college and non-college youth. Journal of Studies on Alcohol, 65, 477-489.

Fontaine, A., Azouvi, P., Remy, P., Bussel, B., \& Samson, Y. (1999). Functional anatomy of neuropsychological deficits after severe traumatic brain injury. Neurology, 10, 19631968.

Franken, I. H. A., \& Muris, P. (2006). Gray's impulsivity dimension: A distinction between Reward Sensitivity versus Rash Impulsiveness. Personality and Individual Differences, 40, 1337-1347. 
Giancola, P. R., Zeichner, A., Yarnell, J. E., and Dickson, K. E. (1996). Relation between executive cognitive functioning and the adverse consequences of alcohol use in social drinkers. Alcoholism: Clinical and Experimental Research, 20(6), 1094-1098.

Grace, J., \& Malloy, P. F. (2001). Frontal Systems Behavior Scale. Professional manual. Lutz, FL: Psychological Assessment Resources, Inc.

Hair, P., \& Hampson, S. E. (2006). The role of impulsivity in predicting maladaptive behaviour among female students. Personality and Individual Differences, 40, 943-952.

Hughes, S. O., Power, T. G., \& Francis, D. G. (1992). Defining patterns of drinking in adolescence: A cluster analytic approach. Journal of Studies on Alcohol, 53(1), 40-47.

Kambouropoulos, N., \& Staiger, P. K. (2004). Reactivity to alcohol-related cues: Relationship among cue type, motivational processes and personality. Psychology of Addictive Behaviours, 18(3), 275-283.

Kane, T. A., Loxton, N. J., Staiger, P. K., \& Dawe, S. (2004). Does the tendency to act impulsively underlie binge eating and alcohol use problems? An empirical investigation. Personality and Individual Differences, 36, 83-94.

Karam, E., Kypri, K., \& Salamoun, M. (2007). Alcohol use among college students: An international perspective. Current Opinion in Psychiatry, 20(3), 213-221.

Kokavec, A., \& Crowe, S. F. (1999). A comparison of cognitive performance in binge versus regular chronic alcohol misusers. Alcohol \& Alcoholism, 34(4), 601-608.

Kypri, K., Cronin, M., \& Wright, C. (2005). Do university students drink more hazardously than their non-student peers? Addiction, 100(5), 713-714.

Langlet, J. D., Kypri, K., \& Stephenson, S. (2003). Second-hand effects of alcohol use among university students: Computerized survey. British Medical Journal, 327, 1023-1024. 
Loxton, N. J., \& Dawe, S. (2001). Alcohol abuse and dysfunctional eating in adolescent girls: The influence of individual differences in sensitivity to reward and punishment. International Journal of Eating Disorders, 29, 455-462.

Lyvers, M. (2000). "Loss of control” in alcoholism and drug addiction: A neuroscientific interpretation. Experimental and Clinical Psychopharmacology, 8, 225-249.

Malloy, P.F., \& Boyle, P.A. (2005). Apathy and its treatment in Alzheimer's disease and other dementias. Psychiatric Times, 23(13), 235-240.

Mazas, C. A., Finn, P. R., \& Steinmetz, J. E. (2000). Decision-making biases, antisocial personality, and early-onset alcoholism: Neurobiological, psychosocial, and developmental correlates of drinking. Alcoholism: Clinical and Experimental Research, 24(7), 1036-1040.

McGee, R. \& Kypri, K. (2004). Alcohol-related problems experienced by university students in New Zealand. Australian and New Zealand Journal of Public Health, 28, 321-323.

Modell, J.G., \& Mountz, J.M. (1995). Focal cerebral blood flow change during craving for alcohol as measured by SPECT. Journal of Neuropsychiatry \& Clinical Neurosciences, 7 , $15-22$.

Noel, X., Paternot, J., Van Der Linden, M., Sferrazza, R., Verhas, M., Hanak, C., Kornreich, C., Martin, P., Del Mol, J., Pelc, I., \& Verbanck, P. (2001). Correlation between inhibition, working memory and delimited frontal area blood flow measured by TcBicisate Spect in alcohol-dependent patients. Alcohol \& Alcoholism, 36(6), 556-563.

Norton, L. E., Malloy, P. F., and Salloway, S. (2001). The impact of behavioural symptoms on activities of daily living in patients with dementia. American Journal of Geriatric Psychiatry, 9(1), 41-48. 
Novak, A., Burgess, E. S., Clark, M., Zvolensky, M. J., \& Brown, R. A. (2003). Anxiety sensitivity, self report motives for alcohol and nicotine use, and level of consumption. Journal of Anxiety Disorders, 17(2), 165-180.

Pardo, Y., Aguilar, R., Molnuevo, B., \& Torrubia, R. (2007). Alcohol use as a behavioural sign of disinhibition: Evidence from J.A. Gray's model of personality. Addictive Behaviours, 32, 2398-2403.

Reinert, D. F., \& Allen, J. P. (2007). The Alcohol Use Disorders Identification Test: An update of research findings. Alcoholism: Clinical and Experimental Research, 31(2), 185-199.

Rose, A. K., \& Duka, T. (2007). The influence of alcohol on basic motoric and cognitive disinhibition. Alcohol \& Alcoholism, 42(6), 544-551.

Saunders, J. B., Aasland, O. G., Babor, T. F., De La Fuente, J. R., \& Grant, M. (1993).

Development of the Alcohol Use Disorders Identification Test (AUDIT): WHO

Collaborative Project on Early Detection of Persons with Harmful Alcohol Consumption: II. Addiction, 88, 791-804.

Sava, F. A., \& Sperneac, A. (2006). Sensitivity to reward and sensitivity to punishment rating scales: A validation study on the Romanian population. Personality and Individual Differences, 41, 1445-1456.

Shields, A. L., \& Caruso, J. C. (2004). A reliability induction and reliability generalization study of the CAGE questionnaire. Educational and Psychological Measurement, 64, 254270.

Spinella, M. (2003). Relationship between drug use and prefrontal-associated traits. Addiction Biology, 8, 67-74. 
Sullivan, E. V., Rosenbloom, M. J., \& Pfefferbaum, A. (2000). Pattern of motor and cognitive deficits in detoxified alcoholic men. American Journal of Psychiatry, 24, 611-612.

Tabachnick, B. G., \& Fidell, L. S. (2007). Using multivariate statistics. (5th Ed.). New York: Harper \&Row.

Torrubia, R., Avila, C., Molto, J., \& Caseras, X. (2001). The Sensitivity to Punishment and Sensitivity to Reward Questionnaire (SPSRQ) as a measure of Gray's anxiety and impulsivity dimensions. Personality and Individual Differences, 31, 837-862.

Varma, V. K., Basu, D., Malhotra, A., Sharma, A., \& Matoo, S. K. (1994). Correlates of earlyand late-onset alcohol dependence. Addictive Behaviours, 19(6), 609-619.

Verdejo-Garcia, A., Rivas-Perez, C., Lopez-Torrecillas, F., \& Perez-Garcia, M. (2006). Differential impact of severity of drug use on frontal behavioural symptoms. Addictive Behaviours, 31, 1373-1382.

Williams, A., \& Clark, D. (1998). Alcohol consumption in university students: The role of reason for drinking, coping strategies, expectancies, and personality traits. Addictive Behaviours, 23(3), 371-378. 